\title{
A review on pharmacological treatment of sleep and circadian rhythm disorders
}

\begin{abstract}
Approximately one third of our lives are spent in sleeping. Although largely an enigma, sleep is an important component of human behavior and provides an emotional and physical restoration to the body. Abnormalities in the normal physiology of sleep often cause a sleep complaint (e.g., difficulty falling asleep) or result in a sleep disorder (e.g., sleep apnea, narcolepsy). The prevalence of sleep difficulties in the general population is estimated to be $30 \%$ to $35 \%$. Sleep is complex psycho-physiologic phenomenon that can be documented in sleep laboratory studies. During sleep the brain is extremely active and produces a characteristic pattern of stages, that cycle during the night. There is a wide inter-individual variability in the amount of sleep required per night (i.e., 3 to 10 hours.) A circadian rhythm of sleep and walking is established shortly after birth and changes over the life cycle. A deceleration of the 24 cycle occurs during adolescence and is typified by difficulty falling asleep. The rhythm returns to 24 hours during adulthood and accelerates with increasing age. Thus elderly individuals commonly complain of falling asleep early in the evening and waking early in the morning. Two oscillators with different period lengths control the suprachiasmic nucleus and the other occurs through neurobiologic mechanism. Two peptides, $\delta$-sleep inducing peptide and factor $\mathrm{S}$ appear to be involved in the biochemical regulation. Synchronization of the oscillators with the 24-hour cycle imposed by the earth's rotation requires a routinely occurring zeitgeber (e.g., clock, light, shower, breakfast) to set the internal clock. Practically, young person should awaken at the same time daily to shorten the oscillators, and elderly persons should delay the onset of sleep until a set time to stretch the oscillators. The neurotransmitter norepinephrine is involved in wakefulness and dreaming, while serotonin is active during non-dreaming sleep. The interaction between the cholinergic system and the noradrenergic system also may play a role in sleep. The pharmacological treatment of sleep and circadian rhythm disorders are updated through this review article.
\end{abstract}

Keywords: Synchronization, neurotransmitter, norepinephrine, apnea, zeitgeber, neurobiologic mechanism, suprachiasmic nucleus, inter-individual variability, psychophysiologic, circadian rhythm, gastroesophageal, electrooculogram, electromyogram, elcectroencephalogram, polysomography
Volume 3 Issue I - 2019

\author{
Amrish Kumar,' Vrish Dhwaj Ashwlayan,' \\ Mansi Verma,' 'Vipin Kumar Garg,' Satish \\ Kumar Gupta,' Avnesh Kumar,' Anurag \\ Chaudhary, ' Anjana Sharma,' Sameksha \\ Koul,' Shobhit Kumar,' Anoop Kumar,' \\ Garima Garg,' Nitin Sharma, ${ }^{2}$ Lubhan Singh, ${ }^{3}$ \\ Abhinav Agrawal, ${ }^{4}$ Sandeep Soni, ${ }^{5}$ Harshit \\ Sharma, ${ }^{6}$ Hement Tyagi, ${ }^{7}$ Firoz Khan ${ }^{8}$ \\ 'Department of Pharmaceutical Technology, Meerut Institute of \\ Engineering and Technology, India \\ ${ }^{2}$ Department of Defence Research and Development, Institute \\ of Nuclear Medicine \& Allied Sciences, India \\ ${ }^{3}$ Department of Pharmacy, Kharvel Subharti College of \\ Pharmacy, India \\ ${ }^{4}$ Department of Pharmacy, Raj Kumar Goel Institute of \\ Technology, India \\ ${ }^{5}$ Department of Pharmacy, KIET School of Pharmacy, India \\ ${ }^{6}$ Scientific Analyst, Serdia Pharmaceuticals, India \\ ${ }^{7}$ Department of Biotechnology, Meerut Institute of Engineering \\ and Technology, India \\ ${ }^{8}$ Clinical Research Coordinator, Jubilant Generics, India
}

Correspondence: Amrish Kumar, M. Pharma, Pharmacology, Research Scholar, Department of Pharmaceutical Technology, Meerut Institute of Engineering and Technology NH-58, Meerut, Uttar Pradesh, India, Email amrish.kumar.miet@gmail.com

Received: December II, 2018 | Published: January 04, 2019
Abbreviations: EEG, electroencephalogram; EOG, electrooculogram; EMG, electromyogram; REM, rapid eye movement; NREM, non- rapid eye movement

\section{Introduction}

The standard method for characterization and measuring sleep stages involves the simultaneous monitoring of an electroencephalogram (EEG), electrooculogram (EOG), and electromyogram (EMG). Two electrooculogram one elcectroencephalogram, and one electromyogram are the minimal recordings used in scoring sleep stages. ${ }^{1}$ Commonly measured objective parameters of sleep include the latency to sleep onset (amount of time to fall asleep), number of awakening, number of stage shift during the night, and intensity of rapid eye movement (REM). Polysomography is a procedure employed to measure multiple physiologic parameters during sleep such as airflow of the nose and mouth, respiratory effort, oxygen saturation, periodic leg movements, gross motor activity, erectile impotence, or gastroesophageal reflux. ${ }^{2}$ Wakefulness is characterized by an EEG of low voltage fast activity, random eye movements and blinks, and a high muscle tone. The Two types of sleep are non-REM (NREM) and REM. Sleep stages 1 through 4 are collectively known as non-REM sleep. During NREM sleep skeletal muscle tone and eye movements are low in comparison with wakefulness and both cardiac and respiratory activity occurs at a slow, regular pace. Stage 1 sleep represents a transition between wakefulness and sleep that lasts between 0.5 and 7 minutes, the EEG reveals low voltage ( 3 to $7 \mathrm{~Hz}$ ) and desynchronized activity. Stage 2 sleep is characterized by a low voltage EEG, and frequent "sleep spindles" (10 to $16 \mathrm{~Hz}$ spindle shaped waves) and "K-complexes" (high voltage spikes). Stages 3 and 4 are called delta sleep and consist of high amplitude, slow delta waves. REM sleep is marked by the onset of a low voltage, mixed frequency EEG and bursts of bilaterally conjugate REMs. During REM sleep muscle tone is low, but autonomic fluctuations (e.g., heart rate, perspiration, penile erection) are active. ${ }^{3}$ Dream reports occur in $80 \%$ to $90 \%$ of subjects if awakened during or at the end of a REM period.

\section{A. Sleep cycle}

The architecture of sleep in healthy young adults is cyclic. From wakefulness the passage through Stages 1 to 4 occurs in 45 minutes. Within 90 minutes the first REM period commences and lasts only 5 to 7 minutes. The cycle lasts approximately 80 to 100 minutes and is repeated 4 to 6 times during the night. Most delta sleep occurs during the first half of the night. The initial REM period progressively lengthens to duration of 30 minutes by the end of the night. A typical young adult spends approximately $75 \%$ of the night in NREM sleep 
(50\% in stages 1 and 2, 25\% in delta) and the remainder in REM sleep. In elderly individuals the sleep pattern is altered with a considerable decrease in delta sleep, REM sleep, and total sleep time. Correspondingly, there is an increase in the number of awakenings and total time spent awake at night. The contribution of daytime napping and specific sleep pathology (e.g., sleep apnea, periodic leg movements) to this apparent decrease in sleep is unclear, however, in randomly selected, non complaining, elderly individuals the incidence of sleep pathology may be as high as $40 \% .{ }^{4}$ Table 1 revealed international classification of sleep disorders (Table 1). ${ }^{5}$

Table I International classification of sleep disorders ${ }^{5}$

\begin{tabular}{l}
\hline Classifications \\
\hline Dyssonmias \\
Intrinsic disorder \\
Extrinsic disorder \\
Circardian disorder \\
Parasomnias \\
Arousal disorder \\
Sleep-wake transition disorder \\
Parasomnias associated with REM sleep \\
Other parasomnias \\
Medical/psychiatric sleep disorders \\
Associated with mental disorder \\
Associated with neurologic disorder \\
Associated with other medical disorder \\
Proposed sleep disorders
\end{tabular}

\section{a. Insomnia}

Insomnia is a subjective complaint of difficulty in falling asleep, maintain sleep or of not feeling rested despite a sufficient opportunity to sleep. ${ }^{6,7}$ A concurrent disturbance of day time functioning (e.g., decreased concentration, fatigue myalgia) usually accompanies the sleep complaint. Younger individuals usually complain of delays in sleep onset, while older patients complain of nocturnal awakening and shorter time periods of sleep.

\section{Epidemiology}

Insomnia is the most prevalent sleep complaint in the general population and one of the most commonly expressed to the physician. And one of the most commonly expressed to the physician. Up to $50 \%$ of the population report insomnia during their lifetimes. ${ }^{8} \mathrm{~A} 1$ year prevalence study of insomnia in the United States reported that one third of individuals surveyed complained of insomnia and $17 \%$ reported the symptoms to be serious. Recent data from the National Institute of Mental Health Epidemiologic Catchment Area Study indicates that the 6 month prevalence of insomnia, defined as symptoms for 2 weeks, was $10.2 \%$. Females, unemployed, elderly separated or widowed individuals, and those in the lower socioeconomic sector reported significantly higher rates of insomnias. Forty percent of those with insomnia had a concurrent psychiatry disorder (e.g., anxiety, depression, alcohol or substance abuse). Table 2 revealed the aetiology of insomnia (Table 2).
Table 2 Aetiolgy of insomnia 9

\begin{tabular}{l}
\hline Etiology of insomnia \\
\hline Situational \\
Work or financial stress \\
Interpersonal conflicts \\
Major life events \\
Jet lag, shift work \\
Medical \\
Cardiovascular (angina, arrhythmias heart failure) \\
Chronic pain \\
Endocrine disorders (diabetes, hyperthyroidism) \\
Gastrointestinal (GERD, ulcer) \\
Neurologic (delirium, epilepsy) \\
Pregnancy \\
Respiratory \\
Psychiatry \\
Mood disorders (depression, Mania) \\
Anxiety disorder \\
Substance abuse (alcohol, hypnotic withdrawal) \\
Pharmacologically induced \\
Anticonvulsants \\
Central adrenergic blocker \\
Steroids
\end{tabular}

Pharmacological treatment of Insomnia

(Table 3). ${ }^{9}$

Table 3 Hypnotic agents

\begin{tabular}{|c|c|c|}
\hline Generic name & $\begin{array}{l}\text { Trade name } \\
\text { (Manufacturer) }\end{array}$ & $\begin{array}{l}\text { Daily dosage } \\
\text { range (mg) }\end{array}$ \\
\hline \multicolumn{3}{|l|}{ Benzodiazepines } \\
\hline Estazolam & ProSom (Abbott) & $1-2$ \\
\hline Flurazepam & Dalmane (Roche) & $15-30$ \\
\hline Quazepam & Doral (Baker Cummins) & $7.5-15$ \\
\hline Temazepam & Restoril (Sandoz) & $15-30$ \\
\hline Triazolam & Halcion (Upjohn) & $0.125-0.25$ \\
\hline \multicolumn{3}{|l|}{ Barbiturats } \\
\hline Amobarbital & Amytal (Lilly) & $100-200$ \\
\hline Pentobarbital & Nembutal (Abbott) & $100-200$ \\
\hline Secobarbital & Seconal (Lilly) & $100-200$ \\
\hline \multicolumn{3}{|c|}{ Nonbarbiturates nonbenzodiazepines } \\
\hline Chloral hydrate & Noctec (Squibb) & $500-2000$ \\
\hline Ethchlorvynol & Placidyl (Abbott) & $500-1000$ \\
\hline Methyprylon & Nodular (Roche) & $200-400$ \\
\hline \multicolumn{3}{|l|}{ Antihistamines } \\
\hline Diphenhydramine & Benadryl (ParkeDavis) & $25-100$ \\
\hline Doxylamine & Unisom (Leeming) & $25-100$ \\
\hline
\end{tabular}




\section{Benzodiazeipine therapy}

In the United States five BZs are marketed with a therapeutic indication for insomnia, however, other BZs also are effective. Flurazepam was the first BZ introduced as a hypnotic in 1970. Triazolam is currently the most widely prescribed BZ hypnotic. The benzodiazepine marketed as hypnotic in abroad include brotizolam, flunitrazepam, loprazolam, lormetazepam, midazolam, and nitrazepam. The benzodiazepine may relieve insomnia by reducing the latency to sleep onset and number of awakening, and increasing the total sleep time. BZs decrease the duration of stages 1 and 4 sleep, and increase stage 2 sleep. Unlike the barbiturates, BZs do not decrease REM sleep to cause a severe R\&M withdrawal syndrome. ${ }^{10}$ Table 4 revealed pharmacokinetic of benzodiazepine hypnotic agent (Table 4).

Table 4 Pharmacokinetic of benzodiazepine hypnotic agents

\begin{tabular}{lllll}
\hline Generic name & $\begin{array}{l}\text { Peak plasma } \\
\text { level (h) }\end{array}$ & $\begin{array}{l}\text { Elimination half-life } \\
\text { parent (h) }\end{array}$ & Metabolic pathway & $\begin{array}{l}\text { Clinically significant } \\
\text { metabolites }\end{array}$ \\
\hline Estazolam & 2 & $12-15$ & Oxidation & - \\
Flurazepam & 1 & 8 & Oxidation & Hydroxyethylflurazepam \\
Quazepam & 2 & 39 & $\begin{array}{l}\text { Oxidation } \\
\text { Conjugation }\end{array}$ & N-DAF $^{\mathrm{a}}$ \\
Temazepam & 1.5 & $10-15$ & Oxidation & - \\
Triazolam & 1 & 2 & & - \\
\hline
\end{tabular}

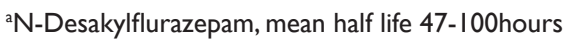

\section{b. Narcolepsy}

Narcolepsy is chronic disease that typically begins before the age of 25 years. About $0.5 \%$ of the adult population are narcoleptics, with men and women being equally affected. There appears to be a genetic predetermination for narcolepsy manifested at puberty, $10 \%$ to $50 \%$ of narcoleptics have a first degree relative with the disorder. ${ }^{11}$ An association between the human leukocyte antigen (HLA)-DR2 and narcolepsy has been identified. The essential feature of narcolepsy is excessive daytime sleepiness with sleep attacks that may last up to 30 minutes. Individuals often complain of hypersomnia, fatigue, impaired performance, and disturbed nighttime sleep. Excessive daytime sleepiness occurs before the second decade of life and the auxiliary symptoms (cataplexy, hypnagogic, hallucinations, sleep paralysis) appear several years later. Cataplexy occurs in $70 \%$ to $80 \%$ of narcoleptics and is characterized by brief episodes (up to 2 minute) of muscle weakness and/or paralysis that may cause the individual to collapse while remaining conscious. Cataplectic episodes often are precipitated by emotionally charged stimuli (e.g., laughter, anger, excitement). Sleep paralysis and hypnagogic hallucination occur in $25 \%$ to $50 \%$ of patients. These phenomena manifest during the transition between wakefulness and sleep, and are loss of than 1 minute in duration. Sleep paralysis is a loss of muscle tone while the patient is still awake. Hypnagogic hallucination occurs when falling asleep and are brief dream like experiences with more fragmentation and bizarre features than a typical dream. Sleep laboratory evaluation of the narcoleptic confirms the existence of excessive daytime sleepiness, disturbed night time sleep, and sleep-onset REM periods. The occurrence of sleep paralysis, cataplexy, and sleep onset REM periods has led to the conclusion that narcolepsy represents an abnormality in the regulatory mechanism of REM sleep. It is clear that the etiology is not psychologic or epileptic.

\section{Treatment}

Management of narcolepsy is symptomatic and consists of both non-pharmacologic interventions. Counseling the patient and significant others is essential as family members often think that narcolepsy is voluntary and the patient is lazy and nonproductive. Good sleep habits should be encouraged. If the patient's daily schedule allows, several daytime naps can be of immense benefit. Following a 15 or 20 minute nap, the patient may feel relatively refreshed for several hours. Long term support groups exist locally and nationally for narcoleptics. ${ }^{12}$ Pharmacologic treatment consists of the use of psycho stimulants for excessive daytime sleepiness and antidepressants for excessive daytime sleepiness and antidepressants for cataplexy. ${ }^{13}$ Methylphenitidine possesses a fast onset of effect and duration of 4 to 6 hours. Total daily doses of methylphenidate rarely exceed $60 \mathrm{mg}$. Pemoline has a delayed onset of effect, but its duration is approximately 12 hours. The maximal effect of pemoline may take several weeks. Liver function tests must be monitored during pemoline therapy. Pemoline in doses of 18.75 to $112.5 \mathrm{mg}$, is the drug of choice in children and patients with mild symptoms. Amphetamine are generally avoided because of the risk of abuse, habituation, and tolerance. Imipramine is effective in reducing the cataplexy and sleep paralysis in daily dosage of 10 to $75 \mathrm{mg}$. Protriptyline at daily dosage of 5 to $60 \mathrm{mg}$ may be effective. Antidepressant efficacy may, to some extent, be based on anticholinergic activity. The monoamine oxidase inhibitor antidepressant phenelzine suppresses cataplexy and selegiline improves hypersomnolence.

\section{B. Circadian rhythm disorders}

Two commonly occurring circadian rhythm sleep disorders are jet lag and shift work sleep problems. The etiology of circadian rhythm disorders is a mismatch between an individual's biologic clock and the external time cues of the environment.

\section{a. Jet lag}

Jet lag follows rapid travel over multiple time zones and results in varying degrees and durations of sleep onset or insomnias usually occurs every other night. Sleep disturbances last for 2 to 3 days, but may prevail for 7 to 10 days if time zone changes are 8 to 12 hours. Compared with westward travel, eastward travel is associated with a longer duration of jet lag. Affected individuals also may suffer from decreased performance and alertness, and gastrointestinal disturbances. ${ }^{14}$ Treatment of Jet lag may consist of preventative measures and pharmacologic management. Jet lag can be avoided during coast to coast travel in the United States for durations of stay less than 7 days in a new time zone by adhering to the normal sleep/ wake schedule from home. For longer lengths of stay, adjusting to a westbound time zone can be made by staying up an arising 1 to 2 hours later several days before the trip. Eastbound travelers also can adjust their schedule by retiring and arising earlier. 


\section{b. Shift Work Sleep Problems}

Shift workers comprise approximately $20 \%$ of the work force. Working at night causes a misalignment in the sleep/wake cycle and circadian rhythms associated with a decrease in alertness, performance, and quality of daytime sleep. On nonworking night shift workers experience insomnia. Treatment may consist of recommending a daytime job, extending daytime sleep by sleeping in the afternoon, or scheduling a 2 to 3 hour afternoon nap on days off from work. Hypnotics should be used cautiously, if at all. Daytime doses of triazolam were associated with cognitive performance deflect in shift workers. Scheduled exposure to bright lights at night and darkness during the daytime improves psychologic and behavioral adaptation to night work and daytime sleep. ${ }^{15}$

\section{Parasomnias}

Although a number of miscellaneous parasomnias have been identified, only three will be discussed.

\section{a. Somnambulism}

Somnambulism is generally a delta sleep phenomenon that occurs early in the night. About $15 \%$ of children experienced at least one sleepwalking episode, compared with $2 \%$ to $5 \%$ of the general adult population. The etiology of somnambulism is unknown, but may involve genetic, developmental, organic or psychologic factors. Somnambulism usually begins in late childhood or early adolescence and abates by late adolescence. Although the patient appears to be navigating well during episode, there is a significant impairment of motor skill and reactivity. The patient should not be awakened during the episode because this may cause confusion. ${ }^{16}$ Treatment of somnambulism consist primarily of protecting the individual from injury. Theoretically, sleepwalking may be prevented by suppressing delta sleep. Although BZs suppress delta sleep, the risks of long term continuous exposure of a developing child to delta sleep suppressants is unknown. BZs may be beneficial in adults

\section{b. Night Terrors}

Night terrors are also known as sleep terror it occurs during the first hours of stage 3-4 non-rapid eye movement sleep. ${ }^{17}$ Night terrors occurs in those children who are between 3-12 years, and usually stop during adolescence. In adults, they most commonly occur between the ages of 20 to 30 . Though the frequency and severity vary between individuals, the episodes can occur in intervals of days or weeks, but can also occur over consecutive nights or multiple times in one night, ${ }^{18}$ prevalent in $1 \%$ to $3 \%$ of children and are outgrown by late adolescence. Night terrors are characterized by extreme vocalization, motility, and autonomic changes (e.g., tachycardia, sweating) coupled with severe panic and fear. Recall of frightening content is minimal or absent. Treatment consists of counseling the parents to wait until the disorder is outgrown. As with somnambulism, night terrors occur during delta sleep, BZs may be useful in adults.

\section{c. Nightmares}

The nightmares are also called as a bad dreams, ${ }^{19}$ are an unpleasant dreams that can cause a strong emotional stress to the brain, typically fear but also despair, anxiety and great sadness. However, psychological nomenclature differentiates between nightmares and bad dreams, specifically; people remain asleep during bad dreams whereas nightmares awaken individuals. The lifetime prevalence of nightmares in the general population is $10 \%$. Nightmare is a REM phenomenon and is associated with frequent and elaborate recall of frightening dream content. Less motility and variability in autonomic parameters are present in nightmares than with night terrors. Once REM-suppressant drug withdrawal has been ruled out as a causative factor, the usual treatment consists of psychologic intervention. This may be as simple as a parent providing comfort and reassurance to a child with an occasional nightmare or as complex as intensive psychotherapy for an adult with frequent, highly disturbing nightmares.

\section{Conclusion}

Disturbances of sleep affect approximately one third of the population. Effective management of sleep disturbance is dependent upon a proper diagnosis. Treatment of sleep disorders includes both pharmacologic and non-pharmacologic modalities. Identifiable causes of insomnia should be managed before pharmacologic therapy is considered. BZs are the preferable agents for the short term treatment of insomnia; however their use is contraindicated in sleep apnea. Antidepressants are an alternative for insomnia and effectively manage sleep apnea and symptoms of narcolepsy. The psycho stimulants are treatment for patients with narcolepsy. Parasomnias and circadian rhythm disorders are managed non-pharmacologically.

\section{Acknowledgments}

None.

\section{Conflicts of interest}

The author declares that there is no conflicts of interest.

\section{References}

1. RechtschaffenA, Kales A. A manual of standardized terminology, technique and scoring system for sleep stages of terminology, technique and scoring system for sleep stages of human subjects. Publication 204, Public Health Service publications. Washington, DC, U.S. Government Printing office. 1968.

2. Doghramji K. Causes, pathogenesis and management of sleep disorders. Compr Ther. 1990;16(3):49-59.

3. Roth T, Hajak G, Ustun TB. Consensus for the pharmacological management of insomnia in the new millennium. Int $J$ Clin Pract. 2001;55(1):42-52.

4. Ancoli-Isrel S, Kripke DF, Mason WJ, et al. Sleep apnea and nocturnal myoclonus in a randomly selected elderly population. J Sleep Res. 1986;15(5):101.

5. American Sleep Disorders Centres (ASCD). Diagnostic classification Steering Committee. International Classification of Sleep Disorders. Diagnostic and Codling Manual. American Sleep Disorders Association, 1990.

6. American Psychiatric Association. Sleep disorders. Diagnostic and Statistical Manual of Mental Disorders. American Psychiatric Press. 1987;297-313.

7. Gillin JC, Byerley WF. The diagnosis and management of insomnia. $N$ Engl J Med. 1990;322(4):239-248.

8. Buysse DJ, Reynolds CF. Handbook of Sleep Disorders. New York, Marcel Dekker. 1990;375-433.

9. Kales A, Soldatos CR, Kales JD. Insomnia night terrors, nightmares and enuresis. JAMA Intern Med. 1987(4);106:582-592.

10. Gaillard JM. Neurotransmitters and sleep pharmacology. Handbook of Sleep Disorders. New York, Marcel Dekker. 1990;55-76.

11. Kales A, Vela-Bueno A, Kales JD. Sleep apnea and narcolepsy. Arch Intern Med. 1987;106:434-443. 
12. Wallace DM, Ramos AR, Rundek T. Sleep disorders and stroke. Int J Stroke. 2012;7(3):231-42.

13. Campbell RK. The treatment of narcolepsy and cataplexy. Drug Intell Clin Pharm. 1981;15(4):257-262.

14. Wagner DR. Circadian rhythm sleep disorders. Handbook of sleep Disorders. New York Marcel Dekker. 1990, pp 493-527.

15. Czeisler CA, Johnson MP, Duffy JF, et al. Exposure to bright light and darkness to treat physilolgic maladaptation to night work. $N$ Engl J Med. 1990;322(18):1253-1259.
16. Kales A, Soldatos CR, Kales JD. Sleep disorder. Insomnia, night terrors, nightmares, and enuresis. Arch Intern Med. 1987;106(4):582-592.

17. Hockenbury Don H, Hockenbury Sandra E. Discovering psychology, 5 th ed. New York: Worth Publishers, 2010. p 157.

18. Dagnostic and statistical manual of mental disorders, 4th ed. United States: American psychiatric press inc (DC). 2000.

19. Harper Douglas. "nightmare”. Online Etymology Dictionary. American Psychiatric Association, Diagnostic and Statistical Manual of Mental Disorders. 2000, 4th ed, p. 631 . 